Chapter 21

\title{
Advancements in Transgenic Soy: From Field to Bedside
}

\author{
Laura C. Hudson, Kevin C. Lambirth, \\ Kenneth L. Bost and Kenneth J. Piller \\ Additional information is available at the end of the chapter \\ http://dx.doi.org/10.5772/52467
}

\section{Introduction}

Today biotechnology and the process of genetic modification is emerging and advancing worldwide. At the forefront of this technology is soybean, which has become a popular subject of genetic modification due to its versatility and economical importance as a crop plant. Over 15 years ago the first herbicide resistant soybeans were introduced into the market. By 1997, approximately $8 \%$ of all soybeans cultivated for commercial use in the United States were genetically modified. This trend has grown exponentially and by 2011 the percentage of genetically modified soybean rose to $94 \%$ in the United States and $81 \%$ worldwide. The technology to genetically modify soybean has not only had a huge impact on the commercial agricultural market, but has paved the way to an onset of both traditional and nontraditional uses for soybean as well as opening up many new potential applications for this important crop plant. Soybean has become a popular subject of genetic modification over the past two decades and with the advancement of plant transformation technology, it is now possible to manipulate and or add various traits to soybean.

\section{Soybean transformation}

There are several protocols used to genetically transform plants with either stable or transient expression. Some of the methods include electroporation, silicon carbide fibers, liposome mediated transformation and in planta Agrobacterium-mediated transformation via vacuum infiltration of whole plant. However, many of these methods are not used for soybean transformation because of low transformation efficiencies. Two more commonly used platforms 
that have been successfully optimized for stable soybean transformation include cotyledonary node-Agrobacterium-mediated transformation and somatic embryo-particle-bombardment-mediated transformation.

The Agrobacterium-mediated plant transformation method uses a soil dwelling bacteria species called Agrobacterium tumefaciens to transfer desirable genes into plants. Using this method, a foreign gene can be placed within the T- DNA boarder regions of the bacterial plasmid which then integrates into a host plant's genome [1]. Wounded plant tissue gives off specific phenolic compounds which induce Agrobacterium to express a set of virulence (vir) genes. The expression of the vir genes results in the production of single-stranded DNA that is transferred and integrated into the plant genome.

There are several advantages of Agrobacterium-meditated plant transformation including straight forward methodology, minimal equipment cost, and reliable insertion of a single or a low copy transgene number. The first reported transformation of soybean with an Agrobacterium strain used co-cultivation followed by organogenesis from cotyledonary nodes [2]. This work was followed by using Agrobacterium mediated transformation of immature cotyledons [3], and embryogenic suspension cultures [4]. Since then, several groups have worked to improve these methods, in particular the transformation and regeneration from cotyledonary nodes. Cotyledonary node regions contain axillary meristems at the junction between cotyledon and hypocotyl. Generally, the cotyledonary nodes are pre-wounded and then co-cultivated with Agrobacterium. The axillary meristems proliferate and regenerate through the formation of multiple adventitious shoots on culture medium containing a cytokinin. In the United States, public facilities, including the Plant Transformation Facility at Iowa State University and the Plant Transformation Core Facility at the University of Missouri, provide fee for service genetic transformation of soybean for public research, mainly by cotyledonary node Agrobacterium-mediated transformation.

The other widely used method of soybean transformation is somatic embryo particle bombardment-mediated transformation also called particle bombardment, or biolistic technology. This method directs small tungsten or gold particles coated with the desired genes toward the target plant cells with enough force to penetrate the cell wall and membrane [5]. Once inside the cell the DNA disassociates from the particle and becomes integrated into the plant genome.

The particle bombardment transformation method was first used in soybean in 1988 by McCabe et al., who successfully transformed immature seed meristem [6] and was followed by the transformation of somatic embryonic tissue [7], and apical meristem [8]. Transformation of somatic embryos has been the most successful method and is induced from immature cotyledons cultured on medium containing moderately high concentrations of an auxin. These cotyledons are used to generate proliferative embryogenic cultures and to recover whole plants. A major advantage of the particle bombardment transformation method relative to Agrobacterium-based methods is the removal of biological incompatibilities between tissues of many plant species and the Agrobacterium vector. However, it has been shown that certain genotypes are more susceptible to the formation of proliferative embryogenic tissue than others. Limitations of the bombardment process include the requirement of specialized 
equipment (gene gun), transformation limited to cells at or near the surface, and high copy number events with high levels of recombination which may not be desirable.

The development of soybean transformation methods has paved the way for an extensive amount of research to develop genetically modified soybeans that have been widely adopted for crop improvement purposes. This has been a fast growing field with the addition of many agronomic, nutraceutical, and pharmaceutical traits being developed, the progress of which will be reviewed in this chapter.

\section{Agronomical improvements in soybean}

\subsection{Herbicide tolerance}

In 1970, glyphosate, a broad-spectrum foliar herbicide, was discovered [9]. Glyphosate inhibits 5-enolpyruvylshikimate-3-phosphate synthase (EPSPS), a crucial enzyme of the shikimate biosynthetic pathway that is responsible for the production of several essential aromatic amino acids. Glyphosate was commercially introduced in 1974 and by 1995 use had reached 4.5 million $\mathrm{kg}$ in the United States. Due to popularity and broad use of this herbicide by farmers, glyphosate became a candidate for research in creating herbicide resistant soybeans and has led to one of the most well-known examples of herbicide tolerance: The Roundup Ready ${ }^{\circledR}$ soybean developed by Monsanto. Roundup Ready ${ }^{\circledR}$ soybeans were one of the first examples of a commercially viable transgenic plant. These transgenic soybeans express functional EPSPS providing tolerance to the herbicide glyphosate (Round$\left.\mathrm{up}^{\mathrm{TM}}\right)$. The popularity of these soybeans grew with farmers since Roundup ${ }^{\mathrm{TM}}$ could be applied to a field of Roundup Ready ${ }^{\circledR}$ soybeans to significantly reduce weed populations while leaving the soybean crop unharmed. The development of Roundup Ready ${ }^{\circledR}$ soybeans offered farmers many advantages in a system that was relatively easy to use. The level and consistency of weed control allowed farmers to take advantage of a no-till system, and eliminated the need for cultivation allowing growers to space rows more closely. Narrow row planting results in higher yields due to a more efficient use of space and may result in better weed control, as the canopy closes more quickly providing earlier competition against weeds. In addition, the window of application for Roundup ${ }^{\mathrm{TM}}$ is wider than for other post emergence herbicides currently used in soybeans, both in terms of the stage of soybean growth and the ability to achieve effective control of larger weeds. These factors contributed to the popularity of this weed control technology. At present, approximately $90 \%$ of the soybeans farmed in the United States utilize this technology.

In response to Monsanto's hugely successful Roundup Ready ${ }^{\circledR}$ crops, Bayer Crop Science released its own herbicide tolerant soybean known as Liberty Link ${ }^{\circledR}$ soybean [10]. Liberty Link $^{\circledR}$ soybeans were developed to express a gene derived from the bacteria Streptomyces viridochromogenes called phosphinothricin-N-acetyltransferase (PAT). PAT is a glutamine synthetase inhibitor that binds to glutamate, making plants resistant to the broad-spectrum contact herbicide glufosinate ammonium. This herbicide causes cessation of photosynthesis and plant death by interfering with the biosynthetic pathway of the amino acid glutamine 
and with ammonia detoxification. Glufosinate ammonium is the active ingredient in phosphinothricin herbicides $\left(\right.$ Basta $^{\circledR}$, Ignite ${ }^{\circledast}$, Rely $^{\circledast}$, Liberty $^{\circledR}$, Harvest $^{\circledR}$, and Finale ${ }^{\circledR}$ ) used to control a wide range of weeds after the crop emerges or for total vegetation control on land not used for cultivation. Since glufosinate ammonium-based herbicides function by a different mode of action than glyphosate-based herbicides, the Liberty Link ${ }^{\circledast}$ system provides farmers with an alternative strategy for controlling weeds.

There are several other examples of transgenic soybeans expressing herbicide resistance traits that are in various stages of development. Pioneer has developed a transgenic soybean product that provides tolerance to two different classes of herbicides: glyphosate and acetolactate synthase (ALS)-inhibiting herbicides. These soybean plants express the glyphosate acetyltransferase (GAT4601) and modified version of a soybean acetolactate synthase (GMHRA) proteins. [11]. The GAT4601 protein confers tolerance to glyphosate-containing herbicides by acetylating glyphosate and thereby rendering it non-phytotoxic. The GM-HRA protein confers tolerance to the ALS-inhibiting class of herbicides. The development of GM soybeans with characteristics controlled by multiple genes leading to the expression of two herbicides is a different approach than previous strategies involving single characteristics controlled by a single gene. These genetically modified soybeans express a combination of herbicides with different modes of action. Inherent crop tolerance will enable more effective management of weed populations.

BASF has used a similar method to introduce a soybean that combines herbicide-tolerant soybean varieties with the broad spectrum imidazolinone class of herbicides. These transgenic soybeans contain the csr1-2 gene derived from Arabidopsis thaliana that encodes the imidazolinone-tolerant AHAS-Large subunit (also known as ALS). The AHAS-L subunit interacts with the endogenous soybean small regulatory subunit to form an enzyme complex that catalyzes the first step in the synthesis of branched-chain essential amino acids, valine, leucine, and isoleucine [12]. The AHAS enzymes occur ubiquitously in plants. Imidazolinone herbicides inhibit the native enzymes, resulting in plant death.

To address the potential emergence of other herbicide resistant broadleaf weeds, Monsanto has developed a line of transgenic soybeans that are resistant to treatment with dicamba [13]. Dicamba (3,6-dichloro-2-methoxybenzoic acid) is a low-cost, widely-used, broad leaf herbicide that is environmentally friendly. Soybeans transformed with a genetically engineered bacterial dicambamonooxygenase (DMO) gene were able to inactivate dicamba, making them resistant to this herbicide. Dicamba-resistant soybeans are in the advanced stages of research and development and are predicted to be commercialized soon.

Syngenta and Bayer CropScience are co-developing HPPD-inhibitor tolerant soybeans, a novel herbicide tolerance trait for soy. The event consists of a molecular stack of a gene conferring tolerance to hydroxyphenylpyruvatedioxygenase (HPPD)-inhibiting herbicides as well as a gene for glufosinate tolerance. Inhibition of HPPD stops the catabolic degradation of tyrosine to plastoquinones which is important for photosynthesis, carotenoid biosynthesis, and tocopherol production [14]. This multiple herbicide tolerance stack will enable the use of multiple herbicides and will be an important new tool for soybean growers faced with increasing pressure from resistant weeds. In the future, other innovative molecular 
strategies can be expected to generate genetically modified (GM) soybeans with novel features to combat weeds and enhance weed resistance. These new GM soybeans will reduce environmental contamination risks and reduce costs for consumers and producers.

\subsection{Insect resistance}

Insect pest management through the use of chemicals has brought about considerable protection to crop yields over the past several decades. Unfortunately, extensive and indiscriminate usage of chemical pesticides has resulted in environmental degradation, adverse effects on human health and other organisms, eradication of beneficial insects, and development of pest-resistant insects. As farmers move forward with the objective of achieving greater crop productivity it will be imperative to replace chemical inputs with safer alternatives to manage insect pests in agricultural ecosystems. Within agricultural biotechnology, insect resistance is a prime research area that has potential to improve agricultural productivity and provide much needed alternatives to pesticides while being effective against pests, innocuous to non-target organisms, and cost effective. With the advent of biotechnology, the ability to genetically modify plants for insect resistance on a commercial scale is within reach. One of the most extensively studied traits for insect resistance in soybeans involves the Bt gene.

Bacillus thuringiensis (Bt) is a common bacteria found in the environment. It has been used as a biological control agent against lepidopteran insects for more than 50 years. Bt targets a class of compounds responsible for insecticidal activity known as crystalline proteins, or cry proteins (Cry1), that are highly toxic after ingestion. The mode of action for Cry1 toxins is the disruption of midgut cellular membranes leading to cell death. One of the primary advantages of using Bt genes for insect control in transgenic plants is the specific insecticidal action toward insects from the Lepidoptera order leaving beneficial insects, birds, and mammals unharmed. Thus, the insertion of Bt toxins into plants, by genetic modification, is an attractive model for the creation of insect resistant transgenic crops.

To date, many different plant species have been genetically modified to exhibit insect resistance using Bt. While the Bt trait has been commercialized in corn and cotton, it is still in developmental stages in soybean. Transformation of soybean with Bt to induce resistance to lepidopteron species has been performed for over a decade. By 1994 fertile transformed soybeans containing a synthetic Bt (Cry1Ac) were generated [15, 16]. Stewart et al., used detached leaf bioassays to show that transgenic soybean lines were resistant to multiple soybean pests with less than 3\% leaf defoliation compared to $20 \%$ observed in traditionally bred lepidopteron resistance soybean lines [16].

Other groups have used a similar strategy by evaluating soybeans engineered with Cry1Ac for resistance to lepidopteron species under field conditions. One example compared Bt lines to controls in the field using field cages and artificial infestation with lepidopteron larvae over a three year period [17]. In this case, Bt lines showed up to 9 times less defoliation from pets when compared to control plants. Similarly, Mcpherson and MacRae reported the evaluation of Bt soybean lines for suppression of lepidopteron species in the field over 2 years [18]. In this case, soybean plants expressing Cry1Ac were essentially absent of lepidopteron populations when compared to peak population densities of 20-30 larvae per row 
in control plots. Furthermore, Bt lines showed $<1.5 \%$ defoliation when compared to $53 \%$ defoliation in control plants.

The utility for Bt soybeans has become evident. This has lead to pyramiding strategies using Cry1Ac with native plant resistance genes to increase plant resistance against insect-pests. Several quantitative trait loci (QTLs) from soybean lines have been described as showing antixenosis and antibiosis resistance towards lepidopteron insects $[19,20]$. This work lead to the development of transgenic soybean lines by combining QTLs with synthetic Cry1Ac [21]. In this case, field evaluations and detached leaf bioassays were used to test this multiple resistance gene pyramiding strategy for antibiosis resistance. Based on defoliation in the field, as well as larval weight gain on detached leaves, soybean lines carrying a combination of Cry1Ac and the QTL were significantly more resistant to lepidopteron pests.

While Bt soybean varieties have not been commercialized this body of research has lead Monsanto to the development of soybeans that incorporate the Bt trait stacked with the second generation Roundup Ready germplasm [22]. Bt Roundup Ready 2 Yield seeds are currently in Phase IV trials and are targeted for commercialization in Brazil in 2013. This pyramiding strategy would be the first in-seed insect protection for soybeans and is expected to offer an important technology for farmers who face significant yield loss due to insect damage. Although not universal in its application and total in its protection, Bt will play a central role in protecting the crop from major insect pests.

With the onset and success of Bt crops other avenues have been explored for their possible roles in the development of transgenic insect resistant plants. These approaches include the use of plant defense proteins, lectins, $\alpha$-amylase inhibitors, insect chitinases, and defensins. The development and implementation of engineered insecticidal soybean varieties is currently in its infancy. The incorporation of a multiple gene stacking strategy will also be important in the future development of insect resistant soybeans. Bt, in combination with other biopesticides, has the potential to drastically reduce the consumption of chemical pesticides, however it will be important to continue research and have a development strategy for a future generation of technology, to ensure that insects do not rapidly develop resistance.

\subsection{Disease resistance}

The United States, Brazil, and Argentina are the three major soybean-producing countries in the world where more than $50 \%$ of all soybeans are harvested. Such a geographic distribution facilitates the spread of insect-pests and diseases. Hence, soybean can be attacked by many different pathogens, including bacteria, viruses, fungi, and nematodes. These pathogens and pests can cause damage in seeds, roots, leaves, stems and pods, and usually are tissue-specific. Therefore, disease resistance is another area of great interest for both researchers and farmers.

Disease control management is currently concentrated on agronomic practices, like planting under tillage, use of lodging resistant varieties, wide row planting, and rotation with nonhost crops. Chemical control has poor efficiency because of low penetration and uneven distribution due to an already formed canopy. In addition, chemical application can be 
extremely expensive for farmers and unhealthy for the environment. There has been little success with conventional plant breeding for disease resistance in soybean leaving room for other approaches such as the use of biotechnology to produce genetically modified soybeans that have disease resistance.

\subsubsection{Bacterial}

Bacterial infections are widespread diseases that occur mainly in the mid-to-upper and young leaves of the soybean plant. There are several bacteria which cause disease in soybean resulting in large amounts of yield loss and poor seed quality. While there has been promising research in the development of bacterial disease resistance with the use of biotechnology in other crop plants such as rice, tomato, banana, and tobacco, there has been less research on the development of bacterial disease resistance for soybean. This research may lead to new strategies for the development of bacterial disease resistance in soybean.

\subsubsection{Viral}

The development of transgenic soybean that confers viral resistance has been studied a bit more extensively. Viruses in soybean are global pests. Significant resistance to several viruses in a number of plant species have been achieved through pathogen derived resistance by the use of viral coat proteins which, when expressed in planta, can interfere with viral assembly. This is the same approach that has been used by several groups to develop transgenic viral resistance in soybean. One of the first groups to investigate this approach was Di et al., who produced a soybean that was resistant to bean pod mottle virus (BPMV) [23]. This was done by introducing a BPMV coat protein into the soybean genome. Transgenic events showed complete resistance to BPMV infection. Another study created soybean lines that were resistant to BPMV by inserting a BPMV capsid polyprotien. Events generated in this case were subjected to infectivity assays and not only exhibited resistance to virus infection, but also exhibited systemic infection, showing little to no visible symptoms [24]. Transgenic lines such as these could lead to future commercial cultivars with resistance to BPMV.

The development of soybean mosaic virus (SMV) resistant soybeans is important since SMV is found in all regions where soybean is grown and infection can cause yield loss up to $90 \%$. Despite progress in other important crop plants, efforts to produce transgenic soybeans resistant to SMV have advanced slowly. In order to produce soybean lines that could confer pathogen derived resistance, plants were produced containing a coat protein gene and the 3 UTR from SMV [25]. Coat protein gene transcripts were detected in transgenic lines and two of the soybean lines were highly resistant to infections with the SMV virus. These results represent the first example of stable genetically engineered SMV resistance in soybean.

The sense coat protein gene of soybean dwarf virus (SbDV) was used to acquire SbDV-resistant soybean plants [26]. These insertions were classified into two types: overexpression of SbDV-CP mRNA, or repression accumulation of SbDV-CP mRNA, and siRNA by RNA analysis prior to SbDV inoculation. In both cases, after infection with SbDV, most plants of these transgenic lines remained symptomless, contained little SbDV-specific RNA and ex- 
hibited SbDV-CP-specific siRNA. The possible mechanism of the achieved resistance was thought to be RNA silencing. This same group later used RNA silencing to create resistance for SbDV using inverted repeat-SbDV coat protein (CP) genes spaced by a $\beta$-glucuronidase sequence [27]. Upon infection with virus, transgenic plants showed no symptoms of the disease. Transgenic soybeans were shown to contain SbDV-CP-specific siRNA and little to no SbDV-specific RNA, suggesting that resistance to SbDV was achieved by an RNA silencingmediated process.

\subsubsection{Fungal}

Fungi are the most common soybean pathogens and therefore represent targets for the development of disease resistant transgenic varieties in soybean. One of the more important fungal diseases affecting soybeans grown in the United States and Brazil is Sclerotinia stem rot (SSR) caused by the fungus Sclerotinia sclerotiorum (white mold). This mold has been associated with the presence of oxalic acid (OA). Treatment of plants with OA induced symptoms whereas metabolism of OA is correlated with fungal tolerance. Cunha et al., generated transgenic soybean lines that overexpressed oxalate decarboxylase (OXDC) [28]. When transgenic soybean lines were infected with white mold the disease progression showed significant reduction of severity that correlated with the level of transgene expression. Transgenic events expressing high levels of OXDC showed complete resistance demonstrating the feasibility of this approach.

Much of the research in the development of fungal disease resistance has focused on overexpression of a single gene to confer protection, though such a method favors co-evolution and pathogenic resistance. An alternative strategy taken by Li et al., was to create multigene resistance by overexpressing multiple anti-fungal genes [29]. Two such genes previously shown to be involved with fungal disease resistance are chitinase (CHI) and the barley ribosome-inactivating protein (RIP). While Li et al., successfully produced transgenic soybean overexpressing both traits, transgenic events were not challenged with fungal infection.

An alternative technology has shown promise with controlling fungal infection through the use of single-chain variable fragment ( $\mathrm{scFv}$ ) antibodies. While plants do not produce endogenous antibodies, they can express and correctly assemble antibody fragments. In fact, antibody production in soybean was first demonstrated in 1998 [30]. A similar antibody approach was recently taken by Brar and Bhattacharyya to control Fusarium virguliforme which is responsible for soybean sudden death syndrome (SDS) [31]. Using the pathogenic toxin Tox1 as a target, soybeans were transformed with an antibody gene encoding scFv anti-FvTox1 to create transgenic lines with enhanced foliar SDS resistance compared to control plants. Their results suggest that FvTox1 is a pathogenicity factor for the development of SDS and that expression of a soybean plant scFv antibody can reduce a toxin-induced plant disease. This biotechnology approach may be translational in fighting other plant diseases that are induced by pathogenic toxins.

To date there are no commercially available transgenic soybeans that confer resistance to disease, including fungal pathogens. In 2011 DuPont, Pioneer Hi-Bred, and Evogene an- 
nounced a collaboration to develop soybean varieties displaying in-plant resistance to soybean rust [32]. This is a major step in the direction of creating the first commercially available transgenic soybean variety that is resistant to a fungal pathogen.

\subsubsection{Nematode}

Plant parasitic nematodes are a significant agricultural problem causing major limitations on crop yield and quality. It is estimated that plant parasitic nematodes cause approximately $\$ 157$ billion [USD] in damage worldwide. Current approaches used to combat agricultural losses include the use of nematicides, cultivation techniques, and varieties with natural resistance. Nematicides include some of the most hazardous compounds used in agriculture and alternative control is required due to health and environmental concerns over their use. In soybean, the majority of yield loss can be attributed to infection by nematodes of the genus Meloidogyne and Heterodera commonly referred to as root knot nematodes (RKN) and soybean cyst nematodes (SCN), respectively. RKN and SCN infect plant roots and induce the formation of specialized feeding sites. The establishment and maintenance of feeding sites are crucial to the survival of nematodes making them an obvious target of interest for novel control strategies. One approach that has emerged in recent years is the use of in planta RNA interference (RNAi) to target genes of feeding nematodes. Through biotechnology, plants can be engineered to produce dsRNAs that silence essential nematode genes. Ingestion of plant-derived dsRNAs by the feeding nematode would trigger the RNAi process thereby inactivating targeted genes and preventing or limiting nematode infection. There are numerous genes known to be essential for nematode survival, and they have been the subject of past reviews $[33,34]$. Many of these appear to be candidates for use in an in planta RNAi strategy to control nematode infection.

Steeves et al., was one of the first to demonstrate efficacy of an RNAi-based strategy to control SCN [35]. Transgenic soybeans were generated following transformation with an RNAi expression vector containing inverted repeats of a cDNA clone of the SCN major sperm protein (MSP). RNA silencing was elicited in the cyst nematode following ingestion of dsRNA molecules, and resulted in $\sim 75 \%$ suppression of reproductive capabilities. Several years later Li et al., used RNAi to test potential gene targets known to be involved with nematode reproduction and fitness [36]. Soybean roots expressing small interfering RNAs against the SCN genes Cpn-1, Y25, and Prp-17 showed a significant reduction in transcript levels in nematode feeding sites. Furthermore, nematode suppression levels were similar to those observed with conventional resistance. Recently RNAi was used to disrupt genes involved with RKN gall formation [37]. Genes encoding tyrosine phosphatase (TP) and mitochondrial stress-70 protein precursor (MSP) were stably expressed in soybean roots, and following infection with RKN the number of galls was decreased by $>90 \%$. Nematode growth within roots was measured and the diameter of nematodes inside transformed soybean roots was reduced 5-fold over that of nematodes inside control roots.

Although there are a few cultivars of soybean that have natural resistance to some species of nematode, there are currently no commercially available soybean varieties that offer genetically modified resistance to nematodes. Over the past 10 years, there have been numerous 
candidate genes found within the nematode-plant interaction that hold potential for the development of novel genetically modified soybeans using an RNAi-based strategy. Results from the above studies show the potential of RNAi technology for reducing gall formation, limiting nematode reproduction and infection, and ultimately broadening soybean resistance to SCN and RKN. The production and eventual commercialization of nematode resistant soybean will benefit both producers and consumers by decreasing dependence on hazardous nematacides and increasing overall soy grain yield.

\section{Soybean trait enhancements}

In 2008 Monsanto announced their Sustainable Yield Initiative - a pledge to double the yields of corn, cotton, and soybeans by the year 2030 while simultaneously reducing aggregate key inputs such as water, land, and energy. While this will be an especially difficult task given that the vast majority of high-quality farm land is already in use, several recent reports involving transgenic soybean technologies support the notion that future biotechnological advances will indeed be able to help achieve such goals.

\subsection{Crop yield}

Crop yield is a highly complex trait, and increases in yield have previously been accomplished through a variety of methods involving traditional breeding and modern biotechnology. The introduction of transgenic crops in 1996 helped improve grain yield by protecting plants from insects and disease pathogens that often result in yield pressure if not treated. While new varieties of soybean combine the latest advances in both modern breeding with genetic modification technologies, there continues to be a search for gene-based approaches with potential to increase soy grain yield. Preuss et al., recently performed a largescale screening for such yield increasing genes and reported that constitutive expression of an Arabidopsis thaliana B-box domain gene (BBX32) resulted in plants with increased plant height, node, flower, pod, and total seed number [38]. More importantly, field grown events showed a $5-8 \%$ increase in plant height, $8-10 \%$ increase in pod number, and $11-14 \%$ increase in total yield relative to control plants. It is believed that overexpression of AtBBX32 modulated circadian clock gene transcripts leading to an increase in the duration of reproductive developmental stages (R3 through R7) of the seed which presumably accounted for the increase in seed yield. Over the next decades, it is likely that seed varieties containing these and other yield traits will be commercialized.

\subsection{Drought resistance}

Drought is a major abiotic stress factor since it can greatly impact crop productivity and grain yield. Soybeans have developed several adaptive traits to endure periods of dry weather and drought. Inclusion of these traits into quality germplasm continues to be a major goal of traditional and marker-assisted breeding programs. While the genetic basis of drought tolerance is not well understood, researchers have focused on understanding 
physiological responses associated with drought (i. e. leaf wilting, water use efficiency, nitrogen fixation, and root growth biomass). While overexpression of single downstream gene targets have shown potential for increasing drought tolerance in Arabidopsis and tobacco model systems, the majority of these findings have not yet been translated to major crop species. One exception involves the overexpression of an endoplasmic reticulum-resident molecular chaperone binding protein (BiP) which is believed to regulate $\mathrm{Ca} 2+$ signaling responses. Valente et al., showed that BiP-overexpressing soybean lines exhibited decreases in leaf wilting, leaf water potential, and stomatal closure under reduced and deprived water conditions [39]. Furthermore, transgenic plants showed decreased rates of photosynthesis and transpiration, steady levels of osmolytes and dry root weight, decreased induction of drought-associated mRNAs, and delayed leaf senescence relative to control plants. While overexpression of BiP shows great potential as a target for increasing drought resistance, it will be important to compare grain yields in field-grown transgenic and control lines.

\subsection{Increased oil content}

Over the past decade, there has been a growing trend for industrial applications utilizing soybean oil, and these applications compete with those used for edible consumption. One example is the recent spike in soy-based biodiesel production which consumed just over 1 billion gallons of soybean oil in 2011 compared with 5 million gallons in 2001 [40]. The growing demand for soybean oil has sparked an interest in novel technologies that could be used to increase the relative oil content of soybean seeds. The retooling of soybean metabolism to increase oil content is not a simple task given that the absolute levels of seed oil and seed protein seem to be set. Increasing oil content comes at the expense of decreasing protein content, and vice versa. To date, only a few papers have reported successes in this area, and both involved manipulation of enzymes and substrate pools in the Kennedy pathway which is responsible for the production of triacylglycerols (TAGs) - the major component of soybean seed oil. In 2008, Lardizabel et al., overexpressed fungal diacylglycerolactetyltransferase (DGAT2) in soybean seeds [41]. DGAT2 converts diacylglycerols (DAGs) to TAGs. Transgenic soybeans overexpressing DGAT2 were grown at 63 locations within the United States and Argentina over five growing seasons, and showed a $1.5 \%$ increase in total seed oil with no reduction of seed protein content or yield. In 2009, Rao and Hildebrand overexpressed the yeast sphingolipid compensation (SLC1) protein in soybean seeds [42]. SLC1 has been shown to have lysophosphatidic acid acyltransferase (LPAT) activity which plays a role in the conversion of lysophosphatidic acid to phosphatidic acid, the precursor to DAG in the Kennedy pathway. Overexpression of yeast SLC1 resulted in soybean somatic embryos with 3. $2 \%$ increased oil content and stable transgenic lines with $1.5 \%$ increased oil content in seeds. Given current commodity pricing for soybean oil [43], a 1.5\% increase in oil adds $\sim \$ 1.2$ billion [USD] in value to the United States soybean crop alone. As soybean oil prices rise it is anticipated that other metabolic engineering strategies will be developed and used to obtain similar increases in seed oil content. 


\section{Health and nutrition}

Soybean is considered a dual use crop since it is a valuable source of seed protein and seed oil. Due to consumer health awareness and an increased demand for vegetable oil, much attention has been drawn to seed oil quality and content. To address these needs, efforts have been made with soybean to increase oxidative stability of soybean oil, enhance fatty acid content of oil, and increase total oil content within the seed. Significant progress has been made by breeders to improve overall yield of soybean, however minimal advancements have been made in the development of high-yield germ lines that have a major shift in carbon flux for increased total protein or oil content in the seed. This may be attributed to the inverse correlation between absolute oil and protein contents within soybean seeds. Biotechnology offers new tools for the development of soybeans that have improved oil quality for use in food, feed, and industrial applications. These nutritional enhancements have been achieved by directed modification of fatty acid biosynthesis to alter amounts of fatty acids that naturally occur in soybean, or to produce novel fatty acids. Two fatty acid profiles that have been targeted through genetic strategies include soybeans with low linolenic acid oil content and high oleic acid oil content.

\subsection{Fatty acid content}

Linolenic acid (LA) accounts for 10-13\% of the total fatty acid content of soybean oil. This fatty acid reduces oxidative stability of oil which results in rancidity and decreased shelflife. A family of three desaturase genes (GmFAD3) contribute to LA biosynthesis in soybean. Flores et al., employed a targeted gene silencing approach to suppress the GmFAD3 gene family using a single RNAi construct [44]. The down regulation of this gene family resulted in low linolenic soybeans with LA contents below $2 \%$. Oleic acid (OA) is a pre-cursor of LA and is considered a healthy source of fat. Conventional soybean oil contains $\sim 18 \%$ OA. While high oleic soybean oil has obvious nutritional value, conventional breeding of high oleic soybean lines have not materialized, in part due to the decrease of yield and environmental instability associated with bred traits. The observed yield drag may be attributed to an alteration in the fatty acid profiles within vegetative tissues of soybean. Progress toward decreasing this yield drag has been made by down regulating $\Delta^{12}$ desaturases (FAD2-1A and $-1 B$ ) which converts OA to LA $[45,46]$. Seeds resulting from this genetic approach have an increased OA content to $\sim 80 \%$ without alterations of vegetative tissue fatty acid contents. In combination with high levels of OA, elevated steric acid levels in soybean oil is also desirable to meet needs of confectionary applications, and development of such soybeans may be possible in the future using similar approaches.

Nutritional enhancements such as increased $\omega$-3 fatty acid levels are also desired for human food and animal feed consumption. Two fatty acids, $\gamma$-linolenic acid (GLA) and stearidonic acid (SDA) are of particular interest since they exhibit pharmacological properties and nutritional value, respectively. Sato et al., were successful at increasing these important fatty acids in soybeans by overexpressing a borage $\Delta^{6}$ desaturase which converts LA and $\alpha$-linolenic (ALA) to GLA and SDA, respectively [47]. Field studies with transgenic 
soybean harboring the borage $\Delta^{6}$ desaturase produced GLA to $\sim 27 \%$ and SDA to $\sim 3 \%$ in seed oil [48]. To increase SDA levels in soy, Eckert et al., pyramided the borage $\Delta^{6}$ desaturase with an Arabidopsis $\Delta^{15}$ desaturase which converts LA to ALA [49]. It was reasoned that increased pools of ALA would lead to increased levels of SDA via the $\Delta^{6}$ desaturase. This strategy resulted in soybean lines with $21.6 \%$ SDA when grown under greenhouse conditions. SDA in turn is a precursor for the long chain polyunsaturated fatty acids eicosapentaenoic acid (EPA) and docosahexaenoic acid (DHA). Diets rich in these fatty acids are associated with cardiovascular fitness. While humans and animals possess the enzymatic machinery to convert SDA to EPA and DHA, diets are often supplemented with EPA and DHA derived from fish oil since vascular plants lack the genes for synthesis of these fatty acids. Attempts have been made to assemble these pathways in soybean by using biosynthetic genes from fungi, algae, and protists along with seed specific co-expression of these genes [50]. In such experiments EPA levels approached 20\% and DHA levels represented up to $3 \%$ of total seed fatty acid content in soybean. The production of such important fatty acids has significant market potential, particularly with respect to human, poultry, pet, and aquaculture feed applications.

\subsection{Tocopherols}

Tocopherols are lipid-soluble antioxidants that are extracted during the commercial processing of soybean seeds and add to the stability of the oil. In soybean there are four forms of tocopherols $(\alpha, \beta, \gamma$ and $\delta$, ) classified by the number of methyl groups present on the molecule. These molecules are collectively referred to as vitamin E, with $\delta$ - and $\gamma$-tocopherols being the most predominant forms in seeds. Biotechnological enhancements of tocopherols in soybean have mainly focused on increasing the amounts of $\alpha$-tocopherol since this form has the highest nutritional value. Attempts to increase total levels of tocopherols have only been marginally successful. Examples of studies to increase total levels have focused on upregulating homogentisatephytyltransferase (HPT) activity in seeds. While HPT catalyzes the first step in tocopherol synthesis, overexpressing lines resulted in little increase of tocopherol in transgenic seeds [51,52]. A modest increase in tocopherol content was achieved by the expression of corresponding HPT genes from Arabidopsis and Synechocystis with a strong seed specific promoter [52]. This approach resulted in a 1.5-fold increase of total tocopherol content. This same group also expressed a bacterial chorismatemutase-prephenate dehydrogenase (TYRA) gene with several other enzymes under the control of seed specific promoters and observed a $>10$ - fold increase in total vitamin $\mathrm{E}$ type molecules.

To generate soybean lines with increased levels of tocopherols a more direct approach has involved overexpression of homogentisategeranylgeranlytransferase (HGGT), an enzyme involved in the biosynthesis of tocopherols in monocots. Recently transgenic expression of rice HGGT was expressed in soybean with a seed-specific and constitutive promoter [53]. Transgenic soybean expressing the HGGT gene had significantly higher levels of antioxidant activities and showed enhanced vitamin E levels associated with the presence of all forms of tocopherols, including tocotrienols (with the exception of the $\beta$ - form) which are not found naturally in soybean. 
Enhanced amounts of $\alpha$-tocopherol in soybean have proven easier to metabolically engineer. Expression of genes for two enzymes responsible for methylation of tocopherol head groups (VTE3 and VTE4) from Arabidopsis were co-expressed within the seed and generated plants with $\alpha$-tocopherol levels greater than $90 \%$ of total tocopherol content [54]. The total levels of tocopherol remained the same in these seeds, showing a shift in tocopherol to mainly the $\alpha$ form yielding a 5-fold increase in vitamin E activity. This research can lead to soybean oil with enhanced vitamin $\mathrm{E}$ and more nutritional value for consumers.

\subsection{Dietary amino acids}

Soy is also deficient in several essential dietary amino acids, most notably methionine and cysteine due to their high sulfur contents. Albumins from Brazil nuts, sunflowers, and corn have been expressed in soybean and although they resulted in increased methionine and cysteine levels, they are not adequate enough to avoid supplementation of these amino acids in animal feed and human diets. The physical synthesis of cysteine is carried out by the enzyme O-acetylserinesulfhydrylase (OASS). In an attempt to increase sulfur containing amino acids in soybean, Kim et al., overexpressed cytosolic OASS and found that transgenic seeds contained elevated levels of both protein bound cysteine (58-74\%) and free cysteine $(22-32 \%)$ [55]. Another approach used to increase sulfur amino acid content in soybean is the use of a maize zein gene which gives rise to several species of insoluble proteins containing high levels of methionine. Dinkins et al., overexpressed maize zein in soybean seeds and observed a $12-20 \%$ increase in methionine and $15-35 \%$ increase in cysteine without adverse effects on protein composition [56].

The essential amino acids lysine and threonine have also been explored for their potential to create nutritionally enhanced soybean. An increased level of lysine was observed by genetically engineering the lysine biosynthetic pathway to circumvent the normal feedback regulation of the enzymes aspartokinase and dihydrodipicolinic acid synthase in soybean [57]. In this case, a $>100$-fold increase of free lysine and 5 -fold increase of total seed lysine content was observed. More recently, soybeans showing enhanced threonine levels have been engineered using seed-specific expression of lysine-insensitive variants of aspartate kinases from bacteria [58]. This strategy produced transgenic soybeans with a 100-fold increase in threonine levels and 3.5-fold increase in total free amino acid content without negative impacts on seed morphology or germination. While enhancement of essential amino acids in soybean seeds has clear potential for commercial applications, it will be important to demonstrate that transgenic soybean with increased nutritional enhancement traits also maintain optimal agronomic characteristics when grown in the field under a variety of conditions.

\section{Soybeans as bioreactors for pharmaceuticals}

Recombinant proteins are widely used in medicine, research laboratories, food and nutrition, and play a key role in important agriculture and biopharmaceutical industries. Since the development of recombinant DNA technology in the early 1970's, the commercial pro- 
duction of recombinant proteins has traditionally relied on a variety of protein expression systems, each with intrinsic advantages and disadvantages. Over the years several methods have been used to produce recombinant proteins. Traditionally, prokaryotic systems based on fermentation have been used for the production of biopharmaceuticals and enzymes. The bacteria Escherichia coli, being one of the earliest and most widely used host for this method, has been used in the production of human insulin since the 1970's [59]. Other platforms include the use of fungal cells and yeast, which have been used as an expression system that is able to perform many of the post-translational modifications required by recombinant protein production. Recombinant proteins that require more complex modifications can be produced using insect or mammalian cells, or transgenic animals. However, major disadvantages associated with these platforms include the inability to perform complex post-translational modifications, the alteration in glycosylation patterns affecting protein activity, the high overall cost associated with manufacturing, the potential for contamination, and long time commitments associated with production in transgenic animals making these platforms impractical for the production of most proteins.

Over the past two decades, there has been a push for recombinant protein technologies to move towards more effective expression systems. These systems must be safe, cost-effective, and conducive to post-translational modifications and processing methods on a large scale. Transgenic plants represent an economical system for accurate expression of complex recombinant proteins on a large scale. Plant cells combine the potential for full post translational modifications and correct protein folding with simple growth requirements. The use of plants as a platform for recombinant protein production has a low risk of contamination with prions, viruses, and other pathogens that infect mammalian cells, and therefore offer advantages that are not associated with existing expression systems. An important advantage of plants as a bioreactor is that recombinant proteins and biopharmaceuticals may be expressed in multiple plant organs including seeds which naturally accumulate high amounts of stored proteins. In general, crops that have higher protein content are more cost-effective for molecular farming. Among recombinant systems that utilize seeds, soybeans present an exceptionally high endogenous protein content, which can reach up to $40 \%$ of the dry seed weight. Soybeans are an ideal source of protein for food and feed thus occupying a unique position as a premier target for genetic engineering, and as a platform for the production of recombinant protein. An important characteristic favoring expression in soybean seeds is that these organs have evolved as specialized compartments to store proteins for embryo nutrition. Based on this, soybean seeds offer an environment with metabolic adaptations that permit the stable and long-term storage of proteins, reducing the requirement for sophisticated and expensive conditions for storage. This makes it possible to stockpile harvested seeds so that the downstream processing can be made available based on the demands of the industry. Similarly, soybean seeds provide a compact compartmentalization biomass, which can considerably reduce overall production costs since purification expenses are typically inversely proportional to the final concentration in the plant biomass.

The concept of a soy-derived pharmaceutical was tested back in 1995 when Cho et al., developed a transformation expression cassette using a soybean seed-specific lectin promoter to 
test for potential expression of the $\beta$-glucuronidase reporter gene [60]. This same expression cassette was used to produce bovine $\beta$-casein in soybean which accumulated to $0.1-0.4 \%$ of seed total soluble protein (TSP) [61]. A follow-up paper characterized post-translational processing, subcellular localization to the PSV, and purification of transgenic $\beta$-casein [62]. These proof-of-concept studies showed that a seed-specific promoter could be used to target stable expression of proteins with commercial value in soybean seed.

\subsection{Antibodies}

Monoclonal antibodies (mAbs) have played a major role in the advancement of biotechnology and development of mAb-based therapeutics and diagnostics. Plants have great potential to serve as a platform for the production of antibodies for therapeutic use. One of the first reports of a functional plant-based antibody was developed in soybean. In an effort to explore cost effective methods of mucosal immunoprotection against sexually transmitted diseases, Zeitlin et al., expressed a humanized monoclonal anti-herpes simplex virus 2 (HSV-2) antibody in leaf tissue [63]. That study compared purified soy-derived and mammalian cellderived HSV-2 mAbs and found that both were similar with respect to stability in human semen and cervical mucus over a 24 hour period. Both antibodies were also able to diffuse in human cervical mucus, and were efficacious in preventing vaginal HSV-2 infection in a murine model.

\subsection{Vaccines}

When plants are mentioned as a platform for the production of pharmaceuticals, the concept of edible vaccines often comes to mind. Edible vaccines are desirable since they would eliminate the use of needles and specialized personnel to administer shots, which may have broad applicability in developing nations. Soybean seeds represent an ideal target for the production of vaccines since soymilk-based formulations are safe and can be easily administered orally. Furthermore, soybean seeds are capable of storing vaccine antigens for many years at ambient temperatures without loss or degradation of the antigen [64-66]. Such features can reduce the need for a cold chain therefore reducing costs.

Vaccines that can be administered at mucosal surfaces offer systemic immunity. Subunit antigens used to vaccinate orally or nasally are often ineffective and require formulation with a mucosal adjuvant for increased efficacy. The heat labile toxin (LT) of E. coli is comprised of a single A subunit (LTA) and pentameric B subunit (LTB) and has been shown to act as both a strong mucosal adjuvant as well as an antigen [67]. Moravec et al., targeted LTB expression to the endoplasmic reticulum of seed storage parenchyma cells where it accumulated to levels up to $2.4 \%$ of seed TSP [68]. Mice orally immunized with seed extracts containing LTB induced both, systemic IgG and mucosal IgA anti-LTB antibody responses. The soybean derived LTB also increased an antibody response against a co-administered bacterial FimHt antigen by 500 -fold demonstrating that soy-derived LTB may function as an oral adjuvant.

Several subunit antigens have been expressed in soybeans that are important to the agricultural industry and could lead to effective vaccines. FanC is a specialized adhesion protein 
located on the bacterial surfaces of Enterotoxigenic E. coli (ETEC). K99 and other ETEC strains cause acute diarrhea in humans and livestock and can be severe and even cause death if left untreated. ETEC vaccinations are routinely administered parenterally to pregnant farm animals in order to stimulate systemic immunity and offer protection in newborns. An edible form of this vaccine has the potential to increase efficacy by conferring mucosal immunity at sites of pathogen invasion. Piller et al., constitutively overexpressed the bacterial FanC antigen in soybeans and reported stable accumulation to levels representing $\sim 0.4 \%$ TSP in both leaves and seeds [69]. Mice immunized with adjuvanted soymilk formulations containing FanC elicited FanC-specific systemic and cellular immune responses demonstrating immunogenicity of the soy-derived antigen.

In another study a soybean-based vaccine was developed against the virus that causes porcine reproductive and respiratory syndrome (PRRS) [70]. PRRS is a serious health problem among breeding swine herds and the current vaccine is not efficacious when applied in the field. Vimolmangkang et al., overexpressed a nucleocapsid protein (PRRSV-ORF7) that accumuolated to $0.64 \%$ of seed TSP. Intragastric immunization of mice with transgenic seed extract, in the absence of adjuvant, induced specific humoral and mucosal immune responses against PRRSV-ORF7 [70].

\subsection{Therapeutics}

Protein therapeutic use is limited by the shortfalls in manufacturing capacity and the high cost of production. While an aging population is a key driver of the protein therapeutics market, the potential for future growth is dependent largely on the industry overcoming drug delivery challenges and cost issues. Plants are cost-effective systems that excel at producing complex therapeutic proteins and therefore could help address some of these issues. The high protein content of soybean seeds, low costs associated with growth, simplified purification methods, and safety, make soybean a unique platform for the production of protein-based therapeutics.

Russell et al., expressed human growth hormone (hGH) in soybean with transformation cassettes using both the constitutive $35 \mathrm{~S}$ promoter as well as a soybean seed-specific promoter 7S $\beta$-conglycyinin [71]. The resulting expression of hGH, both constitutively and within the seed, was detected at low levels of $0.0008 \%$ TSP. More recently hGH was expressed in soybean seeds with a more effective expression cassette utilizing the 7S $\alpha^{\prime}$ subunit of $\beta$-conglycinin promoter and $\alpha$-coixin signal peptide. In this case, hGH was directed to protein storage vacuoles within the seed and accumulated to $2.9 \%$ TSP. Bioassays demonstrated that the soy-derived hGH was fully active [66]. The cost of recombinant E. coli-derived hGH is still a very expensive therapy. Having such a high level of bioactive hGH protein expression in soybean seeds demonstrates the potential for high-yield production of recombinant proteins in soybean seeds and could lead to reduce costs for large-scale production of therapeutic molecules.

Human basic fibroblast growth factor (bFGF) is another high value therapeutic that has been expressed in soybean seeds [72]. This therapeutic was expressed under the control of the soybean seed specific G1 promoter and endogenous signal sequence from soybean. The 
bFGF protein accumulated to levels of $\sim 2.3 \%$ of seed TSP and biological activity of the transgenic protein was confirmed by its mitogenic activity in mice.

Recombinant expression of Insulin was first reported using E. coli [59] and has since been commercialized. Like many pharmaceuticals derived from other expression systems, the potential for contamination along with high costs associated with production remain considerable for this hormone. To show that a soybean expression system could address some of these issues Cunha et al., used a sorghum $\gamma$-kafirin seed storage protein promoter and $\alpha$ coixin PSV signal peptide to target recombinant proinsulin expression to soybean seeds [65]. Transgenic protein was stably expressed in seeds though accumulation levels were not reported. Transgenic seeds containing proinsulin were stable for up to seven years when stored under ambient storage conditions.

The soybean platform has also been used to produce a therapeutic for reducing systolic blood pressure. Novokinin is a hypotensive peptide that has vasorelaxing activity [73]. Novakinin was expressed in soybean seeds under the control of a modified $\beta$-conglycinin promoter and accumulated to $0.5 \%$ of seed TSP. A purified soy-derived formulation, as well as a less pure defatted flour formulation, was orally administered to groups of spontaneously hypertensive rats. Both the purified and partially purified formulations successfully reduced systolic blood pressure after a single dose [74].

Haemophilia B is a bleeding disorder that results from a deficiency of human coagulation factor IX (hFIX). The current treatment for this disease is intravenous infusion of plasma-derived or recombinant hFIX protein. While this treatment is effective at preventing and arresting hemorrhage, it is very costly and the protein is difficult to produce in large quantities. Using a biolistic transformation approach, hFIX expression was targeted to soybean seeds using the soy 7S promoter and coixin signal peptide [75]. Recombinant hFIX protein accumulated to $0.23 \%$ of seed TSP, and purified protein exhibited blood-clotting activity up to $1.4 \%$ of normal plasma demonstrating functionality and efficacy of the soy-derived protein. The recombinant protein was stable for 6 years when stored at room temperature.

Soybeans are also capable of supporting expression and stable accumulation of large and complex proteins that can be difficult or impossible to express using current expression systems. Human thyroglobulin (hTG) is a $660 \mathrm{kDa}$ homodimeric protein that is used as a protein standard and diagnostic for the detection of thyroid disease. To date, no expression system has been capable of producing a recombinant form of hTG which is likely due to strict requirements for correct post-translational modification and proper folding during protein synthesis. As a result, commercial hTG supplied to manufacturers for their assay kits is derived from cadaver and surgically removed thyroid tissue. The heterogeneity and lack of uniformity of commercially-purified hTG preparations is a major factor of variation between kits of different manufacturers. To explore the potential of soybean as a platform for production of large and complex proteins, Powell et al., used the 7S promoter and endogenous hTG signal peptide to target recombinant expression of hTG to soybean seeds [76]. Transgenic lines showed stable expression of full length hTG dimeric protein over multiple generations, and accumulated the protein to levels approaching $1.5 \%$ of seed TSP. Functionality of soy-derived hTG was demonstrated with commercial ELISA kits developed 
specifically for the detection of hTG in patient sera. The expression of $660 \mathrm{kDa}$ dimerich TG appears to be the largest functional recombinant protein expressed in any plant system to date, and demonstrates the practicality of soy as an alternative system for the expression of proteins that are recalcitrant to expression in traditional systems [76].

Soybeans have a high intrinsic capacity for protein production and storage. Other than variability caused by nutrient modulation or environmental effects, the relative distribution of seed protein is primarily determined by genetics. Several groups have been able to achieve recombinant protein expression in soybean seeds at respectable levels (approaching 3\% of seed TSP). However, knowledge of protein distribution in soybeans may help to further maximize expression levels. Schmidt and Herman tested this theory by overexpressing Green Fluorescent Protein (GFP) in soybeans with a $\beta$-conglycinin suppression background, to observe whether proteome rebalancing would result in a higher GFP yield [77]. They found that the rebalancing of intrinsic proteins could be exploited to obtain protein yields which increased $\sim 4$-fold in suppression backgrounds and approached levels representing $>7 \%$ seed TSP. Thus, proteome rebalancing may represent a strategy that can be used to develop soybean lines capable of producing high levels of recombinant proteins in the future.

\section{Conclusion}

Over the past 25 years, soybean production in the United States has grown by nearly $57 \%$ while during that same period the number of acres used to grow soybeans has increased by only $24 \%$. It is predicted that the worldwide requirement for grain will rise by $40-70 \%$ by the year 2050, driven in large part by the growing world population and the increase in demand for protein-rich diets. Clearly the demand for soybean protein and soybean oil is outpacing grain production, which in turn is outpacing available land for growing soybeans. Over the next 50 years, farmers will need to produce as much food as they did in the previous 10,000 years combined, and with fewer resources. The identification of various agricultural improvements such as herbicide, insect, and disease resistance which will allow farmers to obtain increased yields with reduced environmental inputs will be crucial. Traits that not only increase grain yield, but also improve the absolute levels of soy protein and soy oil within the seed will also be important for producers and consumers worldwide. While soybean is recognized for its high protein content, it is also the most widely grown oil-seed crop in the United States. Enhancing nutritional value of soybean oil will greatly increase the effectiveness and value of soy as a food crop, help meet the needs of a growing population, and improve human health.

Over the past decade, soybean has emerged as an ideal expression platform with potential to address current healthcare needs. These unmet needs include cost-effective alternatives to existing protein-based therapeutics, simplified methods for the administration of therapeutics, and the development of reagents that could lead to better diagnostic assays and novel medical devices. Soybeans are unique with respect to protein expression platforms. They are safe to consume, cost-effective to grow, rich in protein content, and stable for years under 
ambient storage conditions. They have been engineered to express a variety of potential therapeutics, including mAbs, vaccine antigens and adjuvants, hormones, growth factors, and blood-clotting factors. Seed-based expression of $660 \mathrm{kDa}$ homodimerich TG underscores the potential of the soybean system to produce large and complex proteins that cannot be produced in yeast, insect, and mammalian cell cultures. The efficacy of engineered therapeutics in crude soymilk formulations could lead to oral vaccines and therapies that require little, if any, purification from other seed proteins. Reports demonstrating long-term stability of seed-derived therapeutics in the absence of climate control directly address cold chain issues associated with vaccines and other therapeutics. With seed protein levels of $\sim 40 \%$ and transgene expression levels approaching 3\% of TSP, a single soybean plant yielding 300 seeds can produce $>500$ mgs of transgenic protein. To put this in perspective, a single soybean plant can produce 500 doses of a vaccine antigen administered at $1 \mathrm{mg} / \mathrm{ml}$, or alternatively, $\$ 50,000$ [USD] of a therapeutic valued at $\$ 100 / \mathrm{mg}$ [USD].

The use of soybean as a platform for the production of therapeutics represents a technology with the potential to revolutionize our current approaches to healthcare. Harnessing the full potential of the soybean platform will depend on further increasing stable transgene expression levels, developing efficient purification methods, obtaining interest from pharmaceutical partners, and overcoming issues associated with commercialization. The production of vaccines, antibodies, and other therapeutic proteins will undoubtedly continue to develop over the next decade. As biotechnology evolves, so does the role of soybean - from the field to the bedside.

\section{Glossary}

EPSPS:5-enolpyruvylshikimate-3-phosphate synthase

PAT:Phosphinothricin-N-acetyltransferase

ALS:Acetolactate synthase

DMO: Dicambamonooxygenase

HPPD:Hydroxyphenylpyruvatedioxygenase

GM:Genetically modified

$\mathrm{Bt}$ :Bacillus thuringiensis

Cry1:Crystalline proteins

QTL:Quantitative trait loci

BPMV:Bean pod mottle virus

SMV:Soybean mosaic virus

SbDV:Soybean dwarf virus 
SSR:Sclerotinia stem rot

OA:Oxalic acid

OXDC:Oxalate decarboxylase

CHI:Chitinase

RIP:Ribosome-inactivating protein

scFv:Single-chain variable fragment

SDS:Sudden death syndrome

RKN:Root knot nematodes

SCN:Soybean cyst nematodes

RNAi:RNA interference

MSP:Major sperm protein

TP:Tyrosine phosphatase

TAGs:Triacylglycerols

DAGS:Diacylglycerols

SCL1:Yeast sphingolipid compensation protein

LPAT:Lysophosphatidic acid acyltransferase

LA:Linolenic acid

OA:Oleic acid

GLA: $\gamma$-linolenic acid

SDA:Stearidonic acid

ALA: $\alpha$-linolenic

EPA:Eicosapentaenoic acid

DHA:Docosahexaenoic acid

HPT:Homogentisatephytyltransferase

HGGT:Homogentisategeranylgeranlytransferase

OASS:O-acetylserinesulfhydrylase

TSP:Total soluble protein

PSV:Protein storage vacuole

mAbs:Monoclonal antibodies (mAbs)

HSV-2:Herpes simplex virus 2 
LT:Heat labile toxin of E. coli

ETEC:EnterotoxigenicE. coli

PRRS:Porcine reproductive and respiratory syndrome

hGH:Human growth hormone

bFGF:Human basic fibroblast growth factor

hFIX:Human coagulation factor IX

hTG:Human thyroglobulin

GFP:Green Fluorescent Protein

\section{Author details}

Laura C. Hudson, Kevin C. Lambirth, Kenneth L. Bost and Kenneth J. Piller

University of North Carolina at Charlotte and SoyMeds, Inc., USA

\section{References}

[1] Horsch RB, Fry JE, Hoffmann NL, Eichholtz D, Rogers SG, Fraley RT. A simple and general method for transferring genes into plants. Science. 1985;227:1229-1231.

[2] Hinchee MAW, Conner-Ward DV, Newell CA, McDonnell RE, Sato SJ, Gasser CS, Fischhoff DA, Re DB, Fraley RT, Horsch RB. Production of transgenic soybean plants using Agrobacterium-mediated DNA transfer. Nat. Biotechnol. 1988;6:915-922.

[3] Parrott WA, Williams EG, Hildebrand DF, Collins GB. Effect of genotype on somatic embryogenesis from immature cotyledons of soybean. Plant Cell Tissue Organ Cult. 1989;16:15-21.

[4] Trick HN, Finer JJ. Sonication-assisted Agrobacterium mediated transformation of soybean [Glycine max (L.) Merrill] embryogenic suspension culture tissue. Plant Cell Rep. 1998;17:482-488.

[5] Christou P, McCabe DE, Swain WF. Stable transformation of soybean callus by DNAcoated gold particles. Plant Physiol. 1988;87:671-674.

[6] McCabe DE, Swain WF, Martinell BJ, Christou P. Stable transformation of soybean (Glycine max) by particle acceleration. Nat. Biotechnol. 1988;6:923-926.

[7] Finer JJ, McMullen MD. Transformation of soybean via particle bombardment of embryogenic suspension culture tissue. In Vitro Cell. Dev. Biol. 1991;27P:175-182. 
[8] Aragão FJL, Sarokin L, Vianna GR, Rech EL. Selection of transgenic meristematic cells utilizing a herbicidal molecule results in the recovery of fertile transgenic soybean [Glycine $\max (\mathrm{L}$.)Merril] plants at a high frequency. Theor. Appl. Genet. 2000;101:1-6.

[9] Franz JE, Mao MK, Sikorski JA. Glyphosate: A Unique Global Pesticide. Washington, DC: American Chemical Society. 1996.

[10] Bayer Crop Science. http://www.bayercropscience.com.

[11] Mathesius CA, Barnett JF Jr, Cressman RF, Ding J, Carpenter C, Ladics GS, Schmidt J, Layton RJ, Zhang JX, Appenzeller LM, Carlson G, Ballou S, Delaney B. Safety assessment of a modified acetolactate synthase protein (GM-HRA) used as a selectable marker in genetically modified soybeans. RegulToxicolPharmacol. 2009;55(3): 309-320.

[12] Lee YT, Duggleby RG. Mutagenesis studies on the sensitivity of Escherichia coliacetohydroxyacid synthase II to herbicides and valine. Biochem. J. 2000;350:69-73.

[13] Behrens MR, Mutlu N, Chakraborty S, Dumitru R, Jiang WZ, LaVallee BJ, Herman PL, Clemente TE, Weeks DP. Dicamba Resistance: Enlarging and preserving biotechnology-based weed management strategies. Science 25 2007;316(5828):1185-1188.

[14] vanAlmsick A. HPPD-Inhibitors - A proven mode of action as a new hope to solve current weed problems. Outlooks on Pest Management. 2009;20(1);27-30.

[15] Parrott WA, All JN, Adang MJ, Bailey MA, Boerma HR, Stewart CN. Recovery and evaluationof soybean plants transgenic for a Bacillus thuringiensis var. kurstaki insecticide gene. In Vitro Cell. Dev.Biol. 1994;30P:144-149.

[16] Stewart CN, Adang MJ, All JN, Boerma HR, Cardineau G, Tucker D, Parrott WA. Genetic transformation, recovery, and characterization of fertile soybean transgenic for a synthetic Bacillus thuringiensis cryIAc gene. Plant Physiol. 1996; 112(1): 121-129. ।

[17] Walker DR, All JN, Mcpherson RM, Boerma HR, Parrott WA. Field evaluation of soybean engineered with a synthetic cry1Ac transgene for resistance to corn earworm, soybean looper, velvetbean caterpillar (Lepidoptera: Noctuidae),and lesser cornstalk borer (Lepidoptera: Pyralidae). J Econ Entomol. 2000;93(3);613- 622.

[18] Mcpherson RM, MacRae TC. Evaluation of transgenic soybean exhibiting high expression of a synthetic Bacillus thuringiensis cry1A transgene for suppressing lepidopteran population densities and crop injury. J Econ Entomol. 2009;102(4)1640-1648.

[19] Cregan PB, Jarvik T, Bush AL, Shoemaker RC, Lark KG, Kahler AL, Kaya N, vanToai TT, Lohnes DG, Chung J, Specht JE. An integrated genetic linkage map of the soybean genome. Crop Science. 1999;39:1464-1490.

[20] Rector BG, All, JN, Parrott WA, Boerma HR. Quantitative trait loci for antibiosis resistance to corn earworm in soybean. Crop Science. 2000;40:233-238. 
[21] Walker DR, Narvel JM, Boerma HR, All JN, Parrott WA. A QTL that enhances and broadens Bt insect resistance in soybean. TheorAppl Genet. 2004;109(5):1051-1057.

[22] Monsanto. http:// www.monsanto.com.

[23] Di R, Purcell V, Collins GB, Ghabiral SA. Production of transgenic soybean lines expressing the bean pod mottle virus coat protein precursor gene. Plant Cell Reports. 1996;15:746-750.

[24] Reddy MS, Ghabrial SA, Redmond CT, Dinkins RD and Collins GB. Resistance to Bean mod mottle virus in transgenic soybean lines expressing the capsid polyprotien. Phytopathology. 2001;91:831-838.

[25] Wang X, Eggenberger AL, Nutter FW Jr., Hill JH. Pathogen-derived transgenic resistance to soybean mosaic virus in soybean. Molecular Breeding. 2001;8(2):119-127.

[26] Tougou M, Furutani N, Yamagishi N, Shizukawa Y, Takahata Y, Hidaka S. Development of resistant transgenic soybeans with inverted repeat-coat protein genes of soybean dwarf virus. Plant Cell Rep. 2006;25(11):1213-1218.

[27] Tougou M, Yamagishi N, Furutani N, Shizukawa Y, Takahata Y, Hidaka S. Soybean dwarf virus-resistant transgenic soybeans with the sense coat protein gene. Plant Cell Rep. 2007;26(11):1967-1975.

[28] Cunha WG, Tinoco MLP, Pancoti HL, Ribeiro RE, Aragão FJL. High resistance to Sclerotinia sclerotiorum in transgenic soybean plants transformed to express an oxalate decarboxylase gene. Plant Pathology. 2010;59(4):654-660.

[29] Li HY, Zhu YM, Chen Q, Conner RL, Ding XD, Li J, Zhang BB. Production of transgenic soybean plants with two anti-fungal protein genes via Agrobacterium and particle Bombardment. Biologia Plantarum. 2004;48(3):367-374.

[30] Zeitlin L, Olmsted SS, Moench TR, Co MS, Martinell BJ, Paradkar VM, Russell DR, Queen C, Cone RA, Whaley KJ. A humanized monoclonal antibody produced in transgenic plants for immunoprotection of the vagina against genital herpes. Nature Biotechnol. 1998;16:1361-1364.

[31] Brar HK, Bhattacharyya MK. Expression of a single-chain variable-fragment antibody against a Fusarium virguliforme toxin peptide enhances tolerance to sudden death syndrome in transgenic soybean plants. MPMI. 2012;25(6);817-824.

[32] Evogen. http://www.evogene.com/News-Events/

[33] Gheysen G, Vanholme B. RNAi from plants to nematodes. Trends Biotechnol. 2007;25(3):89-92.

[34] Lilley CJ, Bakhetia M, Charlton WL, URWIN PE. Recent progress in the development of RNA interference for plant parasitic nematodes. Molecular Plant Pathology. 2007;8:701-711. 
[35] Steeves RM, Todd TC, Essig JS, Trick HN. Transgenic soybeans expressing siRNAs specific to a major sperm protein gene suppress Heterodera glycines reproduction. Funct Plant Biol. 2006;33:991-999.

[36] Li J, Todd TC, Oakley TR, Lee J, Trick HN. Host-derived suppression of nematode reproductive and fitness genes decreases fecundity of Heterodera glycines Ichinohe. Planta. 2010;232(3):775-85.

[37] Ibrahim HM, Alkharouf NW, Meyer SL, Aly MA, Gamal El-Din Ael K, Hussein EH, Matthews BF. Post-transcriptional gene silencing of root-knot nematode in transformed soybean roots. Exp Parasitol. 2011;127(1):90-99.

[38] Preuss SB, Meister R, Xu Q, Urwin CP, Tripodi FA, et al. Expression of the Arabidopsis thaliana BBX32 gene in soybean increases grain yield. PLoS ONE. 2012;7(2):e30717.

[39] Valente MA, Faria JA, Soares-Ramos JR, Reis PA, Pinheiro GL, Piovesan ND, Morais AT, Menezes CC, Cano MA, Fietto L.G. et al. The ER luminal binding protein (BiP) mediates an increase in drought tolerance in soybean and delays drought-induced leaf senescence in soybean and tobacco J. Exp. Bot. 2009;60(2):533-546.

[40] Soystats. http://www.soystats.com.

[41] Lardizabal K, Effertz R, Levering C, Mai J, Pedroso MC, Jury T, Aasen E, Gruys K, Bennett K.Expression of Umbelopsis ramannianaDGAT2A in seed increases oil in soybean. Plant Physiol. 2008;148:89-96.

[42] Rao, SS, Hildebrand D. Changes in oil content of transgenic soybeans expressing the yeast SLC1 gene. Lipids. 2009;44(10):945-951.

[43] CME Group. http://www.cbot.com.

[44] Flores T, Karpova O, Su X, Zeng P, Bilyeu K, Sleper DA, Nguyen HT, Zhang ZJ. Silencing of GmFAD3 gene by siRNA leads to low alpha-linolenic acids (18:3) of fad3mutant phenotype in soybean [Glycine max (Merr.)]. Transgenic Res. 2008;17(5): 839-850.

[45] Mazur B, Krebbers E, Tingey S. Gene discovery and product development for grain quality traits. Science. 1999;285: 372-375.

[46] Buhr T, Sato S, Ebrahim F, Xing A, Zhou Y, Mathiesen M, Schweiger B, Kinney AJ, Staswick P, Clemente T. Ribozyme termination of RNA transcripts down-regulate seed fatty acid genes in transgenic soybean. Plant J 2002;30:155-163.

[47] Sato S, Xing A, Ye X, Schweiger B, Kinney A, Graef G, Clemente T. Production of glinolenic acid and stearidonic acid in seeds of marker free transgenic soybean. Crop Sci. 2004;44:646-652.

[48] Clement T, and Calhoon EB. Soybean Oil: Genetic Approaches for Modification of Functionality and Total Content. Plant Physiology. 2009;151(3):1030-1040.

[49] Eckert H, LaVallee BJ, Schweiger BJ, Kinney AJ, Cahoon EB, Clemente T. Co-expression of the borage $\Delta^{6}$ desaturase and the Arabidopsis $\Delta^{15}$ desaturase results in high ac- 
cumulation of stearidonic acid in the seeds of transgenic soybean. Planta. 2006;224:1050-1057.

[50] Kinney AJ, Cahoon EB, Damude HG, Hitz WD, Kolar CW, Liu ZB. Production of very long chain polyunsaturated fatty acids in oilseed plants. World Patent Application. 2004.

[51] Savidge B, Weiss JD, Wong YH, Lassner MW, Mitsky TA, Shewmaker CK, Post-Beittenmiller D, Valentin HE. Isolation and characterization of homogentisatephytyltransferase genes from Synechocystis sp. PCC 6803 and Arabidopsis. Plant Physiol. 2002;129: 321-332.

[52] Karunanandaa B, Qi Q, Hao M, Baszis SR, Jensen PK, Wong YH, Jiang J, Venkatramesh M, Gruys KJ, Moshiri F, Post-Beittenmiller D, Weiss JD, Valentin HE. Metabolically engineered oilseed crops with enhanced seed tocopherol. Metab Eng. 2005;7(5-6):384-400.

[53] Kim YH, Lee YY, Kim YH, Choi MS, Jeong KH, Lee SK, Seo MJ, Yun HT, Lee CK, Kim WH, Lee SC, Park SK, Park HM. Antioxidant activity and inhibition of lipid peroxidation in germinating seeds of transgenic soybean expressing OsHGGT. J Agric Food Chem. 2011;59(2):584-591.

[54] Van Eenennaam AL, Lincoln K, Durrett TP, Valentin HE, Shewmaker CK, Thorne GM, Jiang J, Baszis SR, Levering CK, Aasen ED, Hao M, Stein JC, Norris SR, Last RL. Engineering vitamin E content: from Arabidopsis mutant to soy oil. Plant Cell. 2003;15(12):3007-3019.

[55] Kim WS, Chronis D, Juergens M, Schroeder AC, Hyun SW, Jez JM, Krishnan HB. Transgenic soybean plants overexpressing O-acetylserinesulfhydrylase accumulate enhanced levels of cysteine and Bowman-Birk protease inhibitor in seeds. Planta. 2012;235(1):13-23.

[56] Dinkins RD, Reddy MSS, Meurer CA, Yan B, Trick H, Thibaud-Nissen F, Finer JJ, Parrott WA, Collins GB. Increased sulfur amino acids in soybean plants overexpressing the maize 15 kDazein protein. In Vitro Cell. Devel. Biol. Plant. 2001;37(6):742-747.

[57] Falco SC, Guida T, Locke M, Mauvais J, Sanders C, Ward RT, Webber P. Transgenic canola and soybean seeds with increased lysine. Biotechnology. 1995;13:577-582.

[58] Qi Q, Huang J, Crowley J, Ruschke L, Goldman BS, Wen L, Rapp WD. Metabolically engineered soybean seed with enhanced threonine levels: biochemical characterization and seed-specific expression of lysine-insensitive variants of aspartate kinases from the enteric bacterium Xenorhabdus bovienii. Plant Biotechnol J. 2011;9(2):193-204.

[59] Goeddel DV, Kleid DG, Bolivar F, Heyneker HL, Yansura DG, Crea R, Hirose T, Kraszewski A, Itakura K, Riggs AD. Expression in Escherichia coli of chemically synthesized genes for human insulin. ProcNatlAcadSci U S A. 1979;76(1):106-110. 
[60] Cho MJ, Widholm JM, Vodkin LO. Cassettes for seed-specific expression tested in transformed embryogenie cultures of soybean. Plant Mol. Binl. Reporter. 1995;13:225-269.

[61] Maughan PJ, Philip R, Cho MJ, Widholm JM, Vodkin LO. Biolistic transformation, expression, and inheritance of bovine $\beta$-casein in soybean (Glycine max). In Vitro Cell. Devel. Biol. Plant. 1999;35:344-349.

[62] Philip R, Darnowski DW, Maughan PJ, Vodkin LO. Processing and localization of bovine $\beta$-casein expressed intransgenic soybean seeds under control of a soybean lectin expression cassette. Plant Science. 2001;161:323-333.

[63] Zeitlin L, Olmsted SS, Moench TR, Co MS, Martinell BJ, Paradkar VM, Russell DR, Queen C, Cone RA, Whaley KJ. A humanized monoclonal antibody produced in transgenic plants for immunoprotection of the vagina against genital herpes. Nature Biotechnol. 1998;16:1361-1364.

[64] Oakes, JL, Bost, KL, Piller, KJ. Stability of a soybean seed-derived vaccine antigen following long-term storage, processing and transport in the absence of a cold chain. Journal of the Science of Food and Agriculture. 2009;89(13):2191-2199.

[65] Cunha, NB, Araujo, AC, Leite, A, Murad, AM, Vianna, GR, Rech, EL. Correct targeting of proinsulin in protein storage vacuoles of transgenic soybean seeds. Genet Mol Res. 2010;9(2):1163-1170.

[66] Cunha NB, Murad AM, Cipriano TM, Cla'udia A, Araujo G,.Aragaỡ FJL, Leite A, Vianna GR, McPhee TR, Souza GHMF, Waters MJ, Elı'bio L. Rech. Expression of functional recombinant human growth hormone in transgenic soybean seeds. Transgenic Res. 2011;20:811-826.

[67] Ryan ET, Crean TI, John M, Butterton JR, Clements JD, Calderwood SB. In vivo expression and immunoadjuvancy of a mutant of heat-labile enterotoxin of Escherichia coli in vaccine and vector strains of Vibriocholerae. Infect Immun. 1999;67(4):16941701.

[68] Moravec T, Schmidt MA, Herman EM, Woodford-Thomas T. Production of Escherichia coli heat labile toxin (LT) B subunit in soybean seed and analysis of its immunogenicity as an oral vaccine. Vaccine. 2007;19;25(9):1647-1657.

[69] Piller KJ, Clemente TE, Jun SM, Petty CC, Sato S, Pascual DW, Bost KL. Expression and immunogenicity of an Escherichia coli K99 fimbriae subunit antigen in soybean. Planta. 2005;222(1):6-18.

[70] Vimolmangkang S, Gasic K, Soria-Guerra R, Rosales-Mendoza S, Moreno-Fierros L, Korban SS. Expression of the nucleocapsid protein of porcine reproductive and respiratory syndrome virus in soybean seed yields an immunogenic antigenic protein. Planta. 2012;235(3):513-522. 
[71] Russell, DA, Spatola, LA, Dian, T, Paradkar, VM, Dufield, DR, Carroll, JA and Schlittler, MR. Host limits to accurate human growth hormone production in multiple plant systems. Biotechnol. Bioeng. 2005;89:775-782.

[72] Ding SH, Huang LY, Wang YD, Sun HC, Xiang ZH. High-level expression of basic fibroblast growth factor in transgenic soybean seeds and characterization of its biological activity. BiotechnolLett. 2006;28(12):869-875.

[73] MatobaN ,Usui H, Fujita H, Yoshikawa M. A novel anti-hypertensive peptide derived from ovalbumin induces nitric oxide-mediated vasorelaxation in an isolated SHR mesenteric artery FEBS Lett. 1999;452:181-184.

[74] Yamada Y, Nishizawa K, Yokoo M, Zhao H, Onishi K, Teraishi M, Utsumi S, Ishimoto $M$, Yoshikawa M. Anti-hypertensive activity of genetically modified soybean seeds accumulating novokinin. Peptides. 2008;29(3):331-337.

[75] Cunha NB, Murad AM, Ramos GL, Maranhão AQ, Brígido MM, Araújo AC, Lacorte C, Aragão FJ, Covas DT, Fontes AM, Souza GH, Vianna GR, Rech EL. Accumulation of functional recombinant human coagulation factor IX in transgenic soybean seeds. Transgenic Res. 2011;20(4):841-855.

[76] Powell R, Hudson LC, Lambirth KC, Luth D, Wang K, Bost KL, PillerKJ. Recombinant expression of homodimeric $660 \mathrm{kDa}$ human thyroglobulin in soybean seeds: an alternative source of human thyroglobulin. Plant Cell Rep. 2011;30(7):1327-1338.

[77] Schmidt MA, Herman EM. Proteome rebalancing in soybean seeds can be exploited to enhance foreign protein accumulation. Plant Biotechnol J. 2008;6(8):832-842. 